\title{
Die soziale Dimension in der Psychotherapie - eine kritische Bestandsaufnahme
}

\author{
Heiner Keupp · Silke Birgitta Gahleitner
}

Eingegangen: 22. Oktober 2017 / Angenommen: 16. November 2017 / Online publiziert: 13. Dezember 2017 (c) Der/die Autor(en) 2017. Dieser Artikel ist eine Open-Access-Publikation.

Zusammenfassung Der Bedeutungszuwachs der Psychotherapie In den 1980er-Jahren ging einher mit einem politischen Wertewandel der 1960er- und 1970erJahre als Reaktion der ersten Generation auf den Holocaust und Nationalsozialismus. In Abgrenzung zur Kriegsgeneration und zum Wirtschaftswachstum formulierte die heranwachsende Generation „posttraditionelle Werte“. Psychotherapie wurde zu einer soziokulturellen Deutungsmacht. Im Zuge der neoliberalen Globalisierung sind die damit verbundenen Forderungen und Ideen jedoch stark in den Hintergrund getreten. Wie also positioniert sich Psychotherapie und mit ihr der psychosoziale Bereich aktuell? Der Artikel reflektiert nach einem kurzen Rückblick in die Historie den aktuellen Stand und schlussfolgert daraus Aufmerksamkeitslinien für eine Berücksichtigung der sozialen Dimension in der Psychotherapie in der aktuellen Lebenswelt.

Schlüsselwörter Psychotherapie - Psychotherapie und Gesellschaft · Sozialkritik · Soziale Dimension in der Psychotherapie · Psychosoziale Arbeit

\section{The social dimension in psychotherapy - a critical review}

Summary The growth in the importance of psychotherapy in the 1980s was associated with a change in political values that had already occurred in the 1960s and 1970s as a reaction of the first post-war

\section{H. Keupp ( $\bowtie)$}

Ringhofferstraße 34, 85716 Unterschleißheim, Deutschland heinerkeupp@psy.lmu.de

\section{S. B. Gahleitner}

Klinische Psychologie und Sozialarbeit, Psychosoziale Diagnostik und Intervention, Alice Salomon Hochschule, Alice-Salomon-Platz 5, 12627 Berlin, Deutschland generation to the Holocaust and National Socialism. Taking a stand against the war generation and the West German "economic miracle", the new generation adopted what it called "post-traditional" values. Psychotherapy became a force whose interpretations had an impact. However, interest in the demands and ideas associated with them was lost following the rise of neoliberal globalisation. What is the position of psychotherapy and the psychosocial services today? Following a brief historical review the article reflects on the current status of the two disciplines, using both as a basis for proposing how and where we should be directing our attention to include the social dimension of psychotherapy in clients' current lifeworlds.

Keywords Psychotherapy - Psychotherapy and society - Social criticism - Social dimension in psychotherapy $\cdot$ Psychosocial work

\section{Einleitung}

In den 1980er-Jahren begannen in den deutschsprachigen Ländern verschiedene Bemühungen, die wachsende Gruppe der PsychotherapeutInnen als Heilberuf neben der ärztlichen Profession zu etablieren. Der Aufschwung der Psychotherapie stand im Zusammenhang mit einem umfassenden Wertewandel in den Nachkriegsjahrzehnten. Die Kinder der Kriegsgeneration lehnten es ab, entfremdete Tätigkeiten auszuüben, sie orientierten sich in Abgrenzung zu ihren Eltern an „posttraditionellen Werten“ und wollten vor allem ihre eigene Selbstentfaltung vorantreiben und zum Beruf machen. Seit den 1960erJahren erhöht sich die Anzahl der Studierenden und Angehörigen psychosozialer Disziplinen und Professionen exponentiell, eine Steigerungsrate, die nur von der Informatik bzw. den Medienwissenschaften 
erreicht oder übertroffen wurde. Die Psychotherapie wurde zu einer soziokulturellen Deutungsmacht, und es gab kaum ein Thema, bei dem sie nicht ihre interpretatorische Hoheit entfaltete.

Die damit verbundenen „weltverbessernden“ Ideen und sozialpolitischen Forderungen sind jedoch im Zuge der neoliberalen Globalisierung immer mehr in sich zusammengesunken. Zwar ist die Nachfrage nach den Studiengängen ungebremst, wir erleben allerdings eine „Enteignung“ des dadurch akkumulierten Wissens. Das Monopol für psychosoziale Interpretationen und Interventionen liegt schon längst nicht mehr bei FachvertreterInnen. BetriebswirtInnen, ManagementexpertInnen, Coaches, Medienfachleute etc. bieten dies alles ebenso an und haben beachtliche Wirtschaftszweige damit geschaffen. Die weite Landschaft der Psychotherapie hat sich in vielen Varianten von den wissenschaftlich fundierten Bezugsdisziplinen, allen voran der Psychologie, gelöst und scheint dort besonders nachgefragt zu sein, wo sich die Entfernung zu einer akademischen Reflexionskultur deutlich abzeichnet.

Wie also positioniert sich Psychotherapie und mit ihr der psychosoziale Bereich aktuell, und was ist aus der einst so vehement vertretenen sozialen Position geworden? Der Artikel reflektiert nach einem kurzen Rückblick in die Historie den aktuellen Stand und schlussfolgert daraus Aufmerksamkeitslinien, um der sozialen Dimension in der Psychotherapie wieder mehr Raum zu schaffen und Gewicht zu verleihen.

\section{Historie}

Bereits 1985 wurde deutlich, dass auch der Kapitalismus sich nicht in einem endlosen Aufschwung nach oben bewegen kann. Es war ersichtlich, dass ökonomische Krisen sich häufen würden und die relative Stabilität der restaurativen gesellschaftlichen Verhältnisse der Nachkriegsjahrzehnte, die die StudentInnenbewegung in die Revolte trieb, sich auflösen würde. Vor allem jedoch wurde offensichtlich, dass die sozialliberalen Demokratisierungs- und Reformansätze der 1970er-Jahre, die wichtige psychosoziale Projekte und Denkrichtungen ermöglicht hatten, sich nicht weiter fortsetzen würden. Ein tief greifender gesellschaftlicher Strukturwandel setzte ein, der als globalisierter Netzwerkkapitalismus eine Reihe von Veränderungen brachte. Castells hat 1996 deutlich gemacht, dass „die Netzwerkgesellschaft einen qualitativen Wandel in der menschlichen Erfahrung“ (S. 477) mit sich bringt: Ihre Konsequenzen „breiten sich über den gesamten Bereich der menschlichen Aktivität aus und transformieren die Art, wie wir produzieren, konsumieren, managen, organisieren, leben und sterben“ (Castells 1991, S. 138).

Im Anschluss an die Aufbauperiode nach dem Faschismus, die vor allem als relativ krisenfreier ökonomischer Aufschwung (Stichwort „Wirtschaftswunder“) gekennzeichnet war, kam es zu einer ersten heftigen
Wirtschaftskrise mit zunehmender Arbeitslosigkeit. Vor diesem Hintergrund haben sich viele Reformhoffnungen der 1960er- und 1970er-Jahre zunehmend als illusionär erwiesen. Lutz (1984) hat dies als das Erwachen aus einem „kurzen Traum immerwährender Prosperität“ bezeichnet. Die Reformbewegungen wie die Antipsychiatrie oder die Gemeindepsychiatrie sowie die Gemeindepsychologie verlieren an Energie. Dies bedeutete einen großen Bruch in den Denktraditionen. In den 1960er- und vor allem den 1970erJahren sind viele psychosozialen Fachkräfte in der Psychiatriereformbewegung sozialisiert worden (vgl. Keupp 2015). Es ging um die Utopie einer veränderten Gesellschaft, in der Ausgrenzung von Dissens und Differenz überwunden und „Verrücktheit“ und „Anderssein“ nicht mehr als abnormal deklassiert sein könnten. Große Worte wie „Deinstitutionalisierung“ oder „Rekommunalisierung psychischen Leids“ wurden zu Reizworten, weil sie von einem utopischen Überschuss getragen wurden, der mehr wollte als technisch-quantitative Lösungen.

Genau in dieser Zeit entstanden auch allgemeingesellschaftliche Diagnosen der „Erschöpfung utopischer Energien“ (Habermas 1985). Zwar hatte der Wertewandel in den 1970er-Jahren, der sich als eine Abkehr von materiellen und Hinwendung zu postmateriellen Werten vollzog (Inglehart 1977), die vor allem auf Freiheit, Selbstbestimmung und Selbstverwirklichung setzten, einer psychosozialen Reflexionskultur eine enorme Schubkraft verliehen, zugleich jedoch wurde der psychosozialen Reformbewegung eine resignative Desillusionierung attestiert: Es stünden „allenthalben Klagen im Vordergrund. Die Psychiatriereform habe nicht gebracht, was man sich von ihr versprochen habe; sie habe gar nicht stattgefunden; sie sei steckengeblieben, weil es am politischen Willen fehle, sie durchzusetzen“ (Finzen 1994, S. 106). Kritik gab es zudem aus den eigenen Reihen: Zunehmende Entpolitisierung war ein zentraler Topos linker Psychologiekritik. KlientInnen würden letztlich lediglich darin unterstützt, sich an gesellschaftlich fragwürdige Verhältnisse anzupassen, statt ihre Veränderung einzuklagen (u. a. Nagel und Seifert 1979; Szasz 1982 [1978]; Zygowski 1987). Die wichtigste Verunsicherung jedoch kam durch Selbstinitiativen von Betroffenen zustande, sie forderten das Recht auf Einbezug und Selbstorganisation.

\section{Globale und lokale Erschöpfung und Burn-out}

Das heute so prominente Thema Burn-out fand in den 1970er-Jahren erstmals Beachtung. In die deutsche Diskussion fand es unter dem Stichwort der „hilflosen Helfer“ Eingang. Schmidbauer (1977) hatte mit diesem Titel einen Bestseller gelandet; als fünf Jahre später sein zweiter Band zum Thema „Helfen als Beruf“ (Schmidbauer 1982) erschien, waren bereits - wie die Verlagswerbung herausstellte - 100.000 Exemplare verkauft: Ein Treffer, der der triumpha- 
listischen Selbstinszenierung des Berufsstandes im deutschsprachigen Raum den Boden entzog. In den USA hatte Freudenberger (1974) das Thema Burnout zum Gegenstand einer ersten fachlichen Analyse gemacht und die Bücher von Cherniss (1980) und Maslach (1982) zum Stresserleben und Burn-out helfender Berufe sind $\mathrm{zu}$ wichtigen Quellen geworden. In der ersten großen Krise nach einer Phase des ungebremsten Auf- und Ausbaus psychosozialer Dienste war eine Deutungsfolie für die Schwierigkeiten und Nöte des Berufsstandes gegeben.

Der Burn-out-Diskurs nahm an Fahrt auf. Heute gibt es eine schon fast inflationäre Beschäftigung mit dem Thema Burn-out, das im globalen Kapitalismus fast alle Berufsgruppen und auch die privaten Lebenswelten erreicht hat. Die helfenden Berufe rangieren dabei an der Spitze der Erschöpfung. Reicht es in der Situation, uns gegenseitig mehr „Selbstsorge“ oder „Achtsamkeit“ zu empfehlen und entsprechende Kursangebote zu machen? So wichtig das in der Ausbildung von professionellen HelferInnen ist und in allen Coaching- und Supervisionsangeboten vorkommen sollte, bleibt doch letztlich die Notwendigkeit, das psychosoziale Handeln in einen größeren gesellschaftlichen Kontext zu stellen und an der Überwindung der zunehmenden „Gesellschaftsblindheit“ oder „sozialen Amnesie“ der aktuellen psychosozialen Professionalität zu arbeiten. Der globalisierte Netzwerkkapitalismus, den Beck (1986) damals als „Risikogesellschaft“ in die Diskussion gebracht hat, erfordert u. E. andere analytische Zugänge. Im Folgenden soll dies kurz begründet und ausgeführt werden.

Im Zuge kultureller Freisetzungsprozesse aus traditionellen Lebensformen hat sich der Alltag in modernen Gesellschaften gravierend verändert (vgl. Beck 1986). Die in den letzten Jahrzehnten fortgeschrittene Pluralisierung von Selbst- und Weltbildern und die Beschleunigung sozialer und kultureller Wandlungsprozesse erfordern eine hohe Flexibilität (vgl. Sennett 1998) und bedingen eine „lebenslange Aufforderung, sich mit Veränderungen auseinanderzusetzen, Neues hinzu- und Überkommenes zu verlernen: Was früher für die Lerngeschichte zwischen den Generationen galt, wird nun zum Problem der individuellen biographischen Lerngeschichte selbst" (Helsper 2000, S. 22). Kulturelle Deutungsmuster und Normalitätsvorstellungen haben sich zugunsten neuer - zunehmend virtueller - Formen der Identitätsgestaltung und Vernetzung verändert und immense Freiräume geschaffen.

Wer mit einer guten Ressourcenausstattung schnell wechselnde soziale und kulturelle Bedingungen flexibel zu nutzen weiß, sieht sich einem attraktiven Angebot an Lebenswegen und Gestaltungsmöglichkeiten gegenüber. Aber auch vielfältige Übergänge und Brüche sind dabei zu bewältigen (vgl. Gahleitner und Hahn 2012). Diese erfordern flexible Orientierungsund Reflexionsleistungen (vgl. Sennett 1998). Die damit verbundenen Anforderungen bringen für Men- schen, die über wenige Ressourcen verfügen, zahlreiche Risiken und Belastungen (vgl. abermals Beck 1986) mit sich. Die Kluft zwischen Arm und Reich hat sich erheblich vergrößert (vgl. BMAS 2008), Exklusionsdynamiken sind die Folge (vgl. für einen Überblick u. a. Keupp et al. 2010). Benachteiligte und beeinträchtigte Menschen geraten auf diese Weise nicht selten ins Abseits (vgl. ebenda). Resultat ist ein zunehmender Verlust sozialer Einbindung und kultureller Einbettung mit positiven wie negativen Konsequenzen für Entwicklungs-, Sozialisations- und Identitätsprozesse (vgl. Keupp 2012; vgl. auch „DisembeddingProzesse“ nach Giddens 2001 [1999]).

Eine Reihe internationaler Untersuchungen zeigt auf, dass soziale Faktoren die wichtigsten Mediatoren zwischen psychischer wie körperlicher Gesundheit und materieller Benachteiligung darstellen (WHO 2001). Auch „biographische Studien kranker Menschen zeigen sehr deutlich, dass Krankheit nicht nur als körperliche Funktionsstörung oder leiblich erlebtes Leiden vorgetragen wird, sondern als tiefe Erfahrung des Normalitätsverlustes und damit im Kern als soziales Problem“ (Hanses 2005, S. 193). Angesichts der psychischen und körperlichen Beeinträchtigungen von Menschen, die an der Grenze zur Armut leben und weder angemessen von psychotherapeutischen noch von Klinik-Settings erreicht werden (oft auch als „hard to reach“ bezeichnet), greifen auf das Individuum bezogene Konzepte zu kurz (vgl. Gahleitner und Pauls 2010), sondern es braucht Hilfeformen, die als Orientierungsangebote ,zwischen den Anforderungen der gesellschaftlichen Funktionssysteme und den Verarbeitungsmöglichkeiten der individuellen Psyche ... helfen, die Exklusion ... möglichst niedrig zu halten" (Großmaß 2006, S. 5).

Solche und verwandte Überlegungen haben bereits im letzten Jahrhundert zu biopsychosozialen Konzeptbildungen geführt (vgl. Engel 1979 [1977], 1980; Uexküll 1963; erweitert Egger 2005; aktuell im Überblick Gahleitner et al. 2013). Krankheit und Gesundheit werden dabei - entlang salutogenetischer Überlegungen (vgl. dazu Antonovsky 1979) - als nicht lineares, komplexes Geschehen verstanden und weder in einzelne, disziplinär fixierte Bestandteile zerlegt noch als rein biologisches Geschehen konzeptualisiert. Obwohl jedoch das biopsychosoziale Modell in seiner Entstehung sehr begrüßt wurde (vgl. u. a. Lolas 1985), ist es inzwischen in viele widersprüchliche Diskurse zersplittert (vgl. dazu Keupp et al. 2013) und hat an Kraft maßgeblich verloren. Die aktuell laufenden Diskussionen beziehen sich nach wie vor auf „Einzelpathologien“.

Die größten Steigerungsraten gibt es bei der Diagnose Depression. Die Einschätzung, dass die Depression zur Volkskrankheit Nr. 1 wird, legt die Frage nahe, welche Ursachen es dafür geben könnte. Aus Frankreich kam vor einiger Zeit unter dem Titel „Das erschöpfte Selbst“ von Ehrenberg (2004 [1998]) ein wichtiger Beitrag, der eine Brücke zwischen so- 
zialwissenschaftlicher Gegenwartsdeutung und der Zunahme diagnostizierter Depressionen schlägt. Er geht davon aus, dass Subjekte in der globalisierten Gesellschaft ein hohes Maß an Identitätsarbeit leisten müssen (Keupp et al. 2013 [1999]). Die zunehmende Erosion traditioneller Lebenskonzepte, die Erfahrung des „Disembedding“ (Giddens 2001 [1999]), die Notwendigkeit zu mehr Eigenverantwortung und Lebensgestaltung haben Menschen in der Gegenwartsgesellschaft viele Möglichkeiten der Selbstgestaltung verschafft. Zugleich ist aber auch das Risiko des Scheiterns gewachsen. Vor allem die oft nicht ausreichenden psychischen, sozialen und materiellen Ressourcen erhöhen diese Risikolagen. Es handelt sich also nicht um eine „Krankheit der Freiheit“, sondern um die Folgen einer individuellen Verinnerlichung der marktradikalen Freiheitsideologien.

\section{Antworten der Psychotherapie?}

Psychotherapie könnte genau für solche Zusammenhänge eine wichtige seismografische Funktion haben. Sie arbeitet an den Krisen der Subjekte und ist damit konfrontiert, dass ihnen die Ressourcen fehlen, die sie zu ihrer Bewältigung bräuchten. Die Häufung spezifischer Krisen und Störungsbilder verweist aber über das einzelne Subjekt hinaus und macht es erforderlich, den kulturell-gesellschaftlichen Hintergrund zu beleuchten und $\mathrm{zu}$ benennen, der diese Krisen fördert. Die in den letzten Jahrzehnten registrierte $\mathrm{Zu}$ nahme etwa von Depressionen, Burn-out-Erfahrungen, Borderline- oder Essstörungen sind Beispiele für die Notwendigkeit, neben einer psychodiagnostischen auch eine gesellschaftsdiagnostische Einordnung vorzunehmen. Bei vielen der aktuell bedeutsamer werdenden Störungsbilder handelt es sich um Identitätskrisen, die auf veränderte gesellschaftliche Lebensbedingungen im globalisierten Netzwerkkapitalismus verweisen. Diese stellen Anforderungen an die alltägliche Identitätsarbeit dar, mit denen viele Menschen nicht mehr zurechtkommen.

Diese Alltagserfahrungen werden in sozialwissenschaftlichen Gegenwartsanalysen aufgegriffen und auf ihre strukturellen Ursachen bezogen. Habermas z. B. hat 1998 eine aufschlussreiche Gegenwartsdiagnose geliefert, in der deutlich wird, dass der Kapitalismus unmittelbar in die Lebensgestaltung der Subjekte eingreift: „Die Ausweitung von Netzwerken des Waren-, Geld-, Personen- und Nachrichtenverkehrs fördert eine Mobilität, von der eine sprengende Kraft ausgeht ... (Habermas 1998). [D]ie Entbindung aus einer stärker integrierten Lebenswelt entlässt die Einzelnen in die Ambivalenz wachsender Optionsspielräume“ (ebd., S. $126 \mathrm{f}$.). Auch der kürzlich verstorbene Bauman (2003 [2000]) hat uns zur Kenntnis gebracht, dass wir in einer „fluiden Gesellschaft“ oder „liquid modernity“ (ebenda; vgl. auch Bauman 2008 [2007]) leben, in der alles Statische und Stabile zu verabschieden ist. Der neue Menschentypus verfügt nach Lifton (1993) über ein „proteisches“ Selbst, welches zwar nicht zur Authentizität findet, aber von einer fluiden Offenheit ist und jede beliebige Gestalt annehmen kann. Interessanterweise ist bereits von einer „proteischen Karriere“ die Rede (Hall 2002, 2004). Die Ambivalenz der Vorlage aus der griechischen Mythologie wird nicht genutzt, um eine solche Entwicklung kritisch zu reflektieren. Sie wird vielmehr zu einer affirmativen Normalität verklärt.

In seinem Buch „Der flexible Mensch“ liefert Sennett (1998) eine weniger positiv gestimmte Analyse der gegenwärtigen Veränderungen in der Arbeitswelt. Der „Neue Kapitalismus“ überschreitet demnach alle Grenzen, demontiert institutionelle Strukturen, in denen sich für die Beschäftigten Berechenbarkeit, Arbeitsplatzsicherheit und Berufserfahrung sedimentieren konnten. Der flexible Mensch hält sich permanent fit für die Anpassung an neue Marktentwicklungen, er bindet sich nicht zu sehr an Ort und Zeit, um immer neue Gelegenheiten nutzen zu können. Lebenskohärenz ist auf dieser Basis kaum mehr zu gewinnen. Sennett (ebenda) hat erhebliche Zweifel, ob der flexible Mensch menschenmöglich ist. Im Rahmen dieses Deutungsversuchs räumt Sennett dem „Scheitern“ oder der mangelnden kommunikativen Bearbeitung des Scheiterns eine zentrale Bedeutung ein. Dieses Scheitern wird oft nicht verstanden und mit Opfermythen oder mit Feindbildkonstruktionen beantwortet. Aus der Sicht von Sennett kann es nur bewältigt werden, wenn es den Subjekten gelingt, das Gefühl ziellosen inneren Dahintreibens, also die „Drift“ (ebenda, S. 15-38) zu überwinden.

Die großen Gesellschaftsdiagnostiker der Gegenwart sind sich in ihrem Urteil also relativ einig: Die aktuellen gesellschaftlichen Umbrüche gehen ans „Eingemachte“ in der Ökonomie, in der Gesellschaft, in der Kultur, in den privaten Welten und auch an die Identität der Subjekte (vgl. auch entsprechende Veröffentlichungen der kritischen Psychologie wie z. B. Knebel 2015), und das hat auch Konsequenzen für Bindungs- und Bildungsprozesse. Die benannten Erfahrungskomplexe an der Nahtstelle von Subjekt und Gesellschaft zeigen, wie stark sich der gesellschaftliche Zustand in unseren Lebenswelten, in Menschenbildern und in Ideologie schon verankert hat (ebenda; vgl. ausführlich Bruder-Bezzel et al. 2016). Deshalb sehen wir schon oft gar keine Alternativen und arrangieren uns mit dem scheinbar naturhaften Ablauf der Dinge. Und genau in dieser Mischung von ,innerer Kolonisierung“ und dem fatalistischen Arrangement mit der Unabwendbarkeit der gesellschaftlichen Abläufe werden wir immer wieder auch zu Komplizen des Status quo und verlieren die Hoffnung, dass es auch sein könnte, dass man etwas gegen die Verhältnisse unternehmen kann und Utopien motivierende Handlungsqualitäten haben.

Wenn wir diese Spur weiterverfolgen wollen, reicht es offensichtlich nicht, nur über „psychohygienische“ und psychotherapeutische Wege zu reden - so wichtig 
sie sind, wenn Menschen schwere psychische Probleme haben. Es ist notwendig, den gesellschaftlichen Rahmen mit in den Blick zu nehmen und danach $\mathrm{zu}$ fragen, wie er einerseits den einzelnen Menschen mit Erwartungen und Ansprüchen fordert und zunehmend überfordert und andererseits die „vereinzelten Einzelnen“ damit alleine lässt. Welche Möglichkeiten also haben wir, um hilfreiche Angebote zu kreieren, die uns Wege aus der erschöpften Gesellschaft weisen könnten, die Zukunftsfähigkeit versprechen, und welche Aufgaben kann psychosoziale Praxis bei einer solchen Zielvorstellung übernehmen?

- Zunächst brauchen wir (wieder) eine umfassendere kritische Auseinandersetzung mit dem neoliberalen Menschenbild des „modularen Menschen“, der ein „Wesen mit mobilen, disponiblen und austauschbaren Qualitäten darstellt“ (Bauman 1999 [1977], S. 158). Zugleich brauchen wir eine „Kultur des Scheiterns“, weil Scheitern vermehrt zu unserer Erfahrung gehört, weil es die Basis für Reflexion und Lernprozesse ist, die Chance zum Neuanfang enthält und weil Scheitern ein Tabu ist. Unsere Kultur wird zunehmend eine „Winner“-Kultur, sie will vor allem Sieger- und Erfolgsgeschichten hören, und sie verdrängt die andere Seite der Medaille.

- Psychotherapie kann und soll dafür Gesellschaftsdiagnostik betreiben und diese im öffentlichen Raum kommunizieren: Die in den privatisierten und individualisierten Problem- und Leidenszuständen der Subjekte enthaltenen gesellschaftlichen Hintergründe kann man entschlüsseln und sichtbar machen. Ottomeyer (2011) zeigt diese Herausforderung am Beispiel des psychotherapeutischen Umgangs mit Trauma über die letzten Jahrzehnte hinweg auf: „Der aus Verdrängung und Verleugnung vorübergehend aufgetauchte existenzielle Schrecken und der Protest gegen die Unmenschlichkeit ... traten zugunsten einer Orientierung an psychiatrisch-medizinischen Manualen und Kassenrichtlinien, an verfeinerten diagnostischen Rastern, an wissenschaftlichen Karrieren, Publikationsund Kongressritualen - teilweise in Richtung auf einen subjektfernen Scientismus - immer mehr in den Hintergrund“ (ebenda, S. 4). Ein kritischerer Umgang mit solchen und ähnlichen Phänomenen wäre Voraussetzung für eine sinnvolle Prävention und Gesundheitsförderung.

- Dazu gehört unmittelbar, dass Psychotherapie ihr Rollenverständnis nicht auf eine operative Dienstleistung reduzieren lässt, wie es die RCT-Forschung mit ihrem Bezug auf Symptomverbesserung nahelegt. Sie benötigt eine Vorstellung davon, für welche Gesellschaft sie mit ihren Kompetenzen einsteht. Will sie Individuen anpassungsfähiger und -bereiter machen, und will sie deren Fitness steigern oder deren „Widerstandsressourcen“ (Antonovsky 1997 [1987]) oder Resilienz fördern, die Distanz und Handlungsfähigkeit gegen die normativen Impe- rative des „unternehmerischen Selbst“ (Bröckling 2007) ermöglichen?

- Psychotherapie benötigt - und dies schulenübergreifend - eine kritische Reflexion ihrer eigenen Menschenbildannahmen. Eine Reihe psychotherapeutischer Technologien im verhaltens- und lösungsorientierten Bereich verdanken sich der Ideologie des Neoliberalismus, sie setzen auf ein Selbstoptimierungsschema, das die/den Einzelne/ $n$ zum Dreh- und Angelpunkt von Selbstinszenierung und Selbstverantwortung macht. Einer ideologisch-theoretischen „Entbettung“ des Subjektes folgt meist eine therapeutisch-praktische. Es wäre den unterschiedlichen psychotherapeutischen Schulen ein Menschenbild zu wünschen, wie es in der Ottawa Charta (WHO 1986) formuliert wurde: „Gesundheit wird von Menschen in ihrer alltäglichen Umwelt geschaffen und gelebt: dort, wo sie spielen, lernen, arbeiten und lieben. Gesundheit entsteht dadurch, dass man sich um sich selbst und für andere sorgt, dass man in die Lage versetzt ist, selber Entscheidungen $\mathrm{zu}$ fällen und eine Kontrolle über die eigenen Lebensumstände auszuüben sowie dadurch, dass die Gesellschaft, in der man lebt, Bedingungen herstellt, die all ihren Bürgern Gesundheit ermöglichen“ (ebenda, S. 5).

- Viel zu lange hat die Psychotherapie dem Thema sozialer Ungleichheit im Zugang zu psychosozialen Ressourcen keine große Beachtung mehr geschenkt, obwohl die Ergebnisse der Forschung keinen Anlass boten, die Relevanz dieser Fragestellung aus dem Blickfeld zu verlieren. In den 1970er- und 1980erJahren wurde die Notwendigkeit gemeindepsychiatrischer Reformmaßnahmen und einer Verbesserung der psychotherapeutischen Basisversorgung unter anderem mit dieser Scherenentwicklung begründet: Die verfügbaren sozialepidemiologischen Daten können bis heute - und dies im steigenden Maße - diese Einschätzung untermauern. Diese individualisierende Verkürzung steht im Widerspruch zu einer wachsenden Ungleichheitsverteilung der materiellen Güter im globalisierten Kapitalismus, und wir haben eindrucksvolle Belege für deren gesundheitspolitische Relevanz.

\section{Ausblick}

Deutlich wird: Um psychosoziale Versorgung im Sozial- wie Gesundheitswesen am „aktuellen Bedarf“ zu orientieren, muss neben einer Reihe anderer Aufgaben auch eine adäquate professionelle Antwort auf die Überforderungen durch psychosoziale Verarbeitungsprozesse aktueller Lebensverhältnisse bereitgestellt werden - und zwar für alle Menschen. Ottomeyer (2011) konstatiert: „Wenn man heute mit traumatisierten Flüchtlingen und Opfern politischer Gewalt arbeitet, hat man unweigerlich mit einer Spannung zwischen Widerstand und Anpassung und dem Ma- 
nagement der eigenen Empörung zu tun“ (S. 3). Die Zeit lässt sich jedoch nicht zurückdrehen, und soziales Engagement muss heute anders aussehen als noch von 30 Jahren. Dennoch ergibt sich mit dem aktuell stattfindenden Generationswechsel ein Verlust bedeutsamer Wissensbestände über die Entstehung und Bedeutung sozialkritischer Ideen und Konzepte. An den Universitäten und Hochschulen wird zunehmend Nachwuchs berufen, der mit den historischen Wurzeln der Psychotherapie und Beratung nicht vertraut ist. Es wird also nicht gelingen, alte Verhältnisse wiederaufzulegen, und dies wäre auch gar nicht wünschenswert. Aber es könnte gelingen, die neue Generation $\mathrm{zu}$ einem Geschichtsbewusstsein $\mathrm{zu}$ motivieren, welches auch neue Entwicklungen in einem anderen und kontextualisierten Licht erscheinen lässt.

Betrachtet man - nur als ein Beispiel von vielen das Konzept der „Verwirklichungschancen“ oder „Capabilities“, wie es von dem Nobelpreisträger Sen (vgl. 2000 [1985]) und seiner Lebenspartnerin Nussbaum (vgl. Nussbaum 1999) entwickelt worden ist, findet man dort z. B. viele Aspekte, die die sozialkritische Haltung verkörpern. Sen (2000 [1985]) knüpft mit seinem Konzept der „Verwirklichungschancen“ z. B. an der Idee der Freiheit und an den gesellschaftlichen Bedingungen an, die zur Realisierung eigener Lebensvorstellungen erforderlich sind. Der Ökonom Sen betont dabei die Bedeutung materieller Grundvoraussetzungen als Verwirklichungschance, aber es kommen weitere Ressourcen hinzu. Über solche und eine Reihe weiterer Konzepte könnte es möglich sein, KlientInnen in ihren Benachteiligungen parteilich-reflexiv $\mathrm{zu}$ begegnen, gesellschaftsdiagnostisch wach $\mathrm{zu}$ bleiben und Wege zu erarbeiten, die ihren Ausgangspunkt und ihre Zielsetzung nicht allein in individuellen Störungen und Lösungsmöglichkeiten begreifen, sondern diese angemessen dorthin kontextualisieren und fokussieren, wo sie ihren Ausgangspunkt genommen haben - im Sinne umfassenderer Lösungsmöglichkeiten als dem Vorhandensein oder Nichtvorhandensein von ICD-Symptomatik.

Interessenkonflikt H. Keupp und S.B. Gahleitner geben an, dass kein Interessenkonflikt besteht.

Open Access Dieser Artikel wird unter der Creative Commons Namensnennung 4.0 International Lizenz (http:// creativecommons.org/licenses/by/4.0/deed.de) veröffentlicht, welche die Nutzung, Vervielfältigung, Bearbeitung, Verbreitung und Wiedergabe in jeglichem Medium und Format erlaubt, sofern Sie den/die ursprünglichen Autor(en) und die Quelle ordnungsgemäß nennen, einen Link zur Creative Commons Lizenz beifügen und angeben, ob Änderungen vorgenommen wurden.

\section{Literatur}

Antonovsky, A. (1979). Health, stress and coping. New perspectives on mental and physical well-being. San Francisco: Jossey-Bass.
Antonovsky, A. (1997). Salutogenese. Zur Entmystifizierung der Gesundheit. Tübingen:DGVT. engl. Orig. 1987

Bauman, Z. (1999). Unbehagen in der Postmoderne. Hamburg: Hamburger Edition. engl. Orig. 1977

Bauman, Z. (2003). Flüchtige Moderne. Frankfurt am Main: Suhrkamp. eng. Orig. 2000

Bauman, Z. (2008). Flüchtige Zeiten. Leben in der Ungewissheit. Hamburg: Hamburger Edition. engl. Orig. 2007

Beck, U. (1986). „Risikogesellschaft“. Auf dem Weg in eine andere Moderne. Frankfurt am Main:Suhrkamp.

Bröckling, U. (2007). Das unternehmerische Selbst. Soziologie einer Subjektivierungsform. Frankfurt am Main: Suhrkamp.

Bruder-Bezzel, A., Bruder, K.-J., \& Münch, K. (Hrsg.). (2016). Neoliberale Identitäten. Der Einfluss der Ökonomisierung auf die Psyche. Gießen: Psychosozial-Verlag.

Bundesministerium für Arbeit und Soziales (BMAS) (2008). Lebenslagen in Deutschland. Der 3. Armuts- und Reichtumsbericht der Bundesregierung. Berlin: BMAS. http:// www.bmas.de/SharedDocs/Downloads/DE/PDFPublikationen-DinA4/forschungsprojekt-a333dritter-armuts-und-reichtumsbericht.pdf. Zugegriffen: 15.10 .2017

Castells, M. (1991). Informatisierte Stadt und soziale Bewegungen. In M. Wentz (Hrsg.), Stadt-Räume. Die Zukunft des Städtischen, (Bd. 2, S. 137-148). Frankfurt am Main: Campus.

Castells, M. (1996). The rise of the network society. The information age: economy, society and culture, Bd. 1. Oxford: Blackwell.

Cherniss, C. (1980). Professional burnout in human service organizations. New York: Praeger.

Egger, J.W. (2005). Das biopsychosoziale Krankheitsmodell. Grundzüge eines wissenschaftlich begründeten ganzheitlichen Verständnisses von Krankheit. Psychologische Medizin, 16(2), 3-12.

Ehrenberg, A. (2004). Das erschöpfte Selbst. Depression und Gesellschaft in der Gegenwart. Frankfurt am Main: Suhrkamp. frz. Orig. 1998

Engel, G.L. (1979). Die Notwendigkeit eines neuen medizinischen Modells: Eine Herausforderung der Biomedizin. InH.Keupp (Hrsg.), Normalität und Abweichung. Fortsetzung einer notwendigen Kontroverse (S.63-85). München: Urban \& Schwarzenberg. engl. Orig. 1977.

Engel, G. L. (1980). The clinical application of the biopsychosocial model. The American Journal of Psychiatry, 137(5), 535-544.

Finzen, A. (1994). Zukünftige Strukturen psychiatrischer Versorgung - Zwischenbilanz und Perspektiven nach zwei Jahrzehnten Psychiatriereform. In F. Reimer (Hrsg.), Vorsorgungsstrukturen in der Psychiatrie(S. 103-110). Berlin: Springer.

Freudenberger, H. J. (1974). Staff burn-out. Journal of Social Issues, 30(1), 159-165.

Gahleitner, S. B., \& Hahn, G. (Hrsg.). (2012). Übergänge gestalten, Lebenskrisen begleiten. Bonn: Psychiatrie-Verlag.

Gahleitner, S. B., \& Pauls, H. (2010). Soziale Arbeit und Psychotherapie. Zum Verhältnis sozialer und psychotherapeutischer Unterstützungen und Hilfen. In W. Thole (Hrsg.), Grundriss Soziale Arbeit. Ein einführendes Handbuch (3. Aufl.S.367-374). Wiesbaden:VS.

Gahleitner, S. B., Hintenberger, G., \& Leitner, A. (2013). Biopsychosozial-zurAktualitätdesinterdisziplinären Modellsin Psychotherapie, Beratung und Supervision. Resonanzen, 1(1), 1-14. http://www.resonanzen-journal.org/article/ view/188/245.Zugegriffen: 15.10.2017. 
Giddens, A. (2001). Entfesselte Welt. Wie die Globalisierung unser Leben verändert. Frankfurt am Main: Suhrkamp. engl. Orig. 1999

Großmaß, R. (2006). Beratung als „neue Profession“-Anstöße und Entwicklungen im Umfeld des Psychotherapeutengesetzes (Deutschland 1999). Vortrag in Basel, September 2006. http://docplayer.org/4697237-Beratung-alsneue-profession-anstoess-e-und-entwicklungen-imumfeld-des-psychotherapeutengesetzes-deutschland1999.html.Zugegriffen 12.12.2017.

Habermas, J. (1985). Die neue Unübersichtlichkeit. Frankfurt am Main:Suhrkamp.

Habermas, J. (1998). Die postnationale Konstellation. Politische Essays. Frankfurt am Main: Suhrkamp.

Hall, D. T. (2002). Careers in and out of organizations. Thousand Oaks:SAGE.

Hall, D. T. (2004). The protean career. A quarter-century journey. Journal of vocational behaviour, 65(1), 1-13.

Hanses, A. (2005). AdressatInnenforschung in der Sozialen Arbeit - Zwischen disziplinärer Grundlegung und Provokation. In C. Schweppe \& W. Thole (Hrsg.), Sozialpädagogik als forschende Disziplin. Theorie, Methode, Empirie(S. 185-200). Weinheim: Juventa.

Helsper, W. (2000). Pädagogisches Handeln in den Antimonien der Moderne. In H.-H. Krüger \& W. Helsper (Hrsg.), Einführung in Grundbegriffe und Grundfragen der Erziehungswissenschaft (4. Aufl. S. 15-34). Opladen: Leske + Budrich.

Inglehart, R. (1977). The silent revolution: changing values and political styles among western publics. Princeton: Princeton University Press.

Keupp, H. (2012). Alltägliche Lebensführung in der fluiden Gesellschaft. In S. B. Gahleitner \& G. Hahn (Hrsg.), Übergänge gestalten, Lebenskrisen begleiten (S. 34-51). Bonn: Psychiatrie-Verlag.

Keupp, H. (2015). Vom utopischen Überschuss zum desillusionierten Realismus. In J. Armbruster, A. Dieterich, D. Hahn \& K. Ratzke (Hrsg.), 40 Jahre Psychiatrie-Enquete. Blick zurück nach vorn (S. 52-64). Köln: Psychiatrie Verlag.

Keupp, H., Ahbe, T., Gmür, W., Höfer, R., Mitzscherlich, B., Kraus, W., \& Straus, F. (2013). Identitätskonstruktionen. Das Patchwork der Identitäten in der Spätmoderne (5. Aufl.). Hamburg: Rowohlt. Erstaufl. 1999

Keupp, H., Rudeck, R., Schröer, H., Seckinger, M., \& Straus, F. (Hrsg.). (2010). Armut und Exklusion. Gemeindepsychologische Analysen und Gegenstrategien. Tübingen:DGVT.

Knebel, L. (2015). Psychotherapie. In M. Allespach \& J. Held (Hrsg.), Handbuch Subjektwissenschaft. Ein emanzipa- torischer Ansatz in Forschung und Praxis (S. 259-284). Frankfurt: Bund-Verlag.

Lifton, R.J. (1993). The protean self. Human resilience in an age of fragmentation. New York: Basic Books.

Lolas, F. (1985). The emergence of the biopsychosocial approach. Amodel for third world health care systems. Social Science and Medicine, 21(12), 1337.

Lutz, B. (1984). Der kurze Traum immerwährender Prosperität. Eine Neuinterpretation der industriell-kapitalistischen Entwicklung im Europa des 20. Jahrhunderts. Frankfurt am Main: Campus.

Maslach, C. (1982). Burnout. The cost of caring. Englewood Cliffs: Prentice Hall.

Nagel, H., \& Seifert, M. (Hrsg.). (1979). Inflation der Therapieformen. Gruppen- und Einzeltherapien in der sozialpädagogischen und klinischen Praxis. Sinn und Unsinn der Psycho-Industrie. Reinbek: Rowohlt.

Nussbaum, M.C. (1999). Gerechtigkeit oder das gute Leben. Frankfurtam Main: Suhrkamp.

Ottomeyer, K. (2011). Traumatherapie zwischen Widerstand und Anpassung. Journal für Psychologie, 19(3), Art. 4. Onlineverfügbar:http://www.journal-fuer-psychologie.de/ index.php/jfp/article/view/89/.Zugegriffen: 09.11.2017.

Schmidbauer, W. (1977). Hilflose Helfer. Über die seelische Problematik der helfenden Berufe. Reinbek: Rowohlt.

Schmidbauer, W. (1982). Helfen als Beruf. Die Ware Nächstenliebe. Reinbek: Rowohlt.

Sen, A. K. (2000). Ökonomie für den Menschen. Wege zu Gerechtigkeit und Solidarität in der Marktwirtschaft. München: Hanser. engl. Orig. 1985

Sennett, R. (1998). Der flexible Mensch. Die Kultur des neuen Kapitalismus. Berlin: Berlin Verlag.

Szasz, T. S. (1982). Der Mythos der Psychotherapie. Wien: Europaverlag. engl. Orig. 1978

v. Uexküll, T. (1963). Grundfragen der psychosomatischen Medizin. Reinbek: Rowohlt.

World Health Organization (WHO) (1986). Ottawa-Charta zur Gesundheitsförderung. Genf:WHO. http://www.euro. who.int/_data/assets/pdf_file/0006/129534/Ottawa_ Charter_G.pdf.Zugegriffen: 15.10.2017

World Health Organization (WHO) (2001). The World Health Report 2001. Mental health: new perspectives, new hope. Genf: WHO. www.who.int/entity/whr/2001/en/whr01_ en.pdf.Zugegriffen: 15.10.2017

Zygowski, H. (Hrsg.). (1987). Psychotherapie und Gesellschaft. Therapeutische Schulen in der Kritik. Reinbek: Rowohlt. 